OPEN ACCESS

Edited by:

Michael James Winkelman, Arizona State University, United States

Reviewed by:

Fabrizio Schifano,

University of Hentfordshire

United Kingdom

Brian D. Earp,

University of Oxford, United Kingdom Ido Hartogsohn,

Bar-Ilan University, Israel

*Correspondence:

Dimitris Repantis

dimitris.repantis@charite.de

Specialty section:

This article was submitted to

Psychopharmacology,

a section of the journal

Frontiers in Psychiatry

Received: 31 March 2021

Accepted: 09 June 2021

Published: 02 August 2021

Citation:

Langlitz N, Dyck E, Scheidegger M

and Repantis D (2021) Moral

Psychopharmacology Needs Moral

Inquiry: The Case of Psychedelics.

Front. Psychiatry 12:680064.

doi: 10.3389/fpsyt.2021.680064

\section{Moral Psychopharmacology Needs Moral Inquiry: The Case of Psychedelics}

\author{
Nicolas Langlitz ${ }^{1}$, Erika Dyck ${ }^{2}$, Milan Scheidegger ${ }^{3}$ and Dimitris Repantis ${ }^{4 *}$ \\ ${ }^{1}$ Department of Anthropology, The New School for Social Research, New York, NY, United States, ${ }^{2}$ Department of History, \\ University of Saskatchewan, Saskatoon, SK, Canada, ${ }^{3}$ Department of Psychiatry, Psychotherapy and Psychosomatics, \\ University Hospital of Psychiatry Zurich, Zurich, Switzerland, ${ }^{4}$ Charité - Universitätsmedizin Berlin, Department of Psychiatry \\ and Psychotherapy, Berlin, Germany
}

The revival of psychedelic research coincided and more recently conjoined with psychopharmacological research on how drugs affect moral judgments and behaviors. This article makes the case for a moral psychopharmacology of psychedelics that examines whether psychedelics serve as non-specific amplifiers that enable subjects to (re-)connect with their values, or whether they promote specific moral-political orientations such as liberal and anti-authoritarian views, as recent psychopharmacological studies suggest. This question gains urgency from the fact that the return of psychedelics from counterculture and underground laboratories to mainstream science and society has been accompanied by a diversification of their users and uses. We propose bringing the pharmacological and neuroscientific literature into a conversation with historical and anthropological scholarship documenting the full spectrum of moral and political views associated with the uses of psychedelics. This paper sheds new light on the cultural plasticity of drug action and has implications for the design of psychedelic pharmacopsychotherapies. It also raises the question of whether other classes of psychoactive drugs have an equally rich moral and political life.

Keywords: psychedelics, hallucinogens, morality and values, extrapharmacological variables, anthropology, ethnography, history, psychotherapy

\section{INTRODUCTION: A NEW FIELD OF RESEARCH}

Since the 2000s, neuropsychopharmacologists have grown interested in the neurochemical foundations of moral behaviors. Experimental paradigms from social psychology and behavioral economics have been used to study the effects of drugs, for example how antidepressants such as citalopram enhanced harm aversion and affected people's inclination to retaliate against unfairness (1), how oxytocin affected one's readiness to trust others (2) and motivated both in-group favoritism and out-group derogation (3), how regular users of stimulants showed less prosocial behavior (4), or how MDMA and LSD increased emotional empathy and altruism (5). Analogous to moral psychology, a field that emerged at about the same time, this rapidly growing body of research gave birth to a new subfield that could aptly be called "moral psychopharmacology." 


\section{THE CONTEXT DEPENDENCE OF PSYCHEDELICS VARIES THEIR MORAL EFFECTS}

In the case of psychedelics, research on their moral effects is of particular importance. Politically, psychedelic drug use is likely to increase and take place in a variety of non-medical contexts as a number of jurisdictions introduce decriminalizing legislation concerning psychedelics. In May 2021, for example, Psilonautica and Drug Science, two UK-based organizations, revealed findings from a study showing that a majority of the British public support the medical use of psychedelics for trauma-based injuries and end-of-life anxiety. American cities like Denver, Oakland and Santa Cruz went further and decriminalized psilocybin, and in 2020 the state of Oregon decriminalized psilocybin and approved it for medical use. The legal prohibition on psychedelics is beginning to change, but its regulatory future remains context specific. Scientifically, the moral psychopharmacology of psychedelics is especially interesting because their effects have long been shown to depend crucially on extra-pharmacological factors. Since the 2010s, what is colloquially called "set and setting," the subject's mindset and their social and physical environment, has attracted renewed interest in the revival of psychedelic research. Carhart-Harris et al. (6) argued that the drugs' $5 \mathrm{HT}_{2 \mathrm{a}}$ receptor agonism renders the psychedelic experience exceptionally sensitive to context, both internal and external. While common psychophysiological effects on serotonin-mediated neurobehavioral circuits may shape some of the cross-cultural similarities in patterns of psychedelic use and experiences, their cultural desirability is mostly shaped by political factors (7). What distinguishes research in this vein from research on the placebo effect is that, in the case of psychedelics, the effects of extra-pharmacological factors appear to be pharmacologically mediated and amplified. It is part of psychedelic drug action to blur the line between the pharmacological and the nonpharmacological, between drug action and social context.

This context-dependence is also of clinical importance as psychedelic-assisted therapy of depression and other psychiatric conditions combines pharmacotherapy with psychotherapy, actively mobilizing extra-pharmacological factors to improve treatment outcomes (even though ongoing randomized controlled trials do not explicitly focus on the psychotherapeutic contribution). The subjective experience shaped by set and setting might not just be epiphenomenal but causally related to the drugs' therapeutic effects (8). Although interactions with other people crucially affect how patients experience the effects of psychedelics, in medical research, it is often a mere afterthought that drug treatments of psychiatric patients have significant implications for their social relationships (9). Outside of a clinical setting, these contextual features will be even more difficult to assess, predict, or control.

This relationship to cultural context opens up a dimension of moral psychopharmacology directly relevant to clinical work, which cannot be guided only by considerations of the normal and the pathological that usually inform medical interventions. Thereby, the question of how drugs affect human relationships and what makes a good relationship comes into the purview of psychiatric drug treatment. In the clinical context, moral psychopharmacology moves from the limited ecological validity of behavioral economic games and moral dilemma experiments to issues that are highly relevant to the moral fabric of everyday life (even if the intricacies of romantic relationships or family life have not figured prominently in moral philosophy). In this respect, the moral psychopharmacology of all drugs that affect social behavior appears significant. Notably, recent studies highlight how psychedelics and empathogens modulate conscious decisionmaking and behavioral orientations in various psycho-socioenvironmental domains $(5,10,11)$. The pronounced contextdependence of psychedelics and their application in the context of different psychotherapeutic approaches or non-clinical uses gives particular urgency to the question of how they affect people's moral judgment and behavior. After all, the moral effects might not be one-dimensional, simply increasing or decreasing regard for others as concerns about moral "enhancement" and "degradation" imply $[(12,13)$, p. 233-35, 261-63]. Instead, the moral effects of psychedelics appear to differ depending on the context of their use and the mind-set of the user.

The successful mainstreaming of psychedelics through medical research has introduced these substances to populations beyond the so-called psychedelic research community. The growing diversity of users and uses broadens the range of extra-pharmacological factors that shape the drugs' moral effects. Historical and ethnographic research can complement contemporary neuropsychopharmacology studies with studies of cultural contexts that examine other sets and settings in relation to drug uses and effects. While members of the American counterculture used LSD, mescaline, and psilocybin as psychopharmacological tools to liberate individuals from the ills of their society, Huichol youth ingested peyote buttons to become full members of their own society $(14,15)$, and Native American Church worshippers consumed peyote to foster indigenous resistance to North American colonialism $(16,17)$. In the 1960s, psychedelics were taken to experience a mystical union that users claimed fostered a sense of universal love, but anthropologists have also described Amazonian societies that used them in rituals to prepare for violent intergroup conflict (18-20). At present, psychedelic therapists and progressive intellectuals suggest that psychedelic experiences could help to work through the cultural trauma of racist discrimination $(21,22)$, and one study found that intense psychedelic experiences predicted liberal political views, openness, and nature relatedness, while negatively predicting authoritarian political views [(23); but see (24)]. At the same time, a budding traditionalist scene finds inspiration in the right-wing writer and psychonaut Ernst Jünger and, according to media reports, psychedelics might also play a role in the political radicalization of far-right groups in the United States (25-27). A moral psychopharmacology of psychedelics needs to explain how pharmacological and extrapharmacological factors interact to produce such seemingly contradictory varieties of psychedelic experience. 


\section{TAKING PSYCHEDELIC RESEARCH BEYOND THE TWO-CULTURE DIVIDE}

A well-established but potentially misleading account of the moral and political versatility of psychedelics draws from an older naturalist ontology that suggests that there is one nature and many cultures that interpret it differently. It has inspired a neat division of labor between the disciplines: neuropsychopharmacologists study the effects of drugs on the brain, cultural anthropologists and historians study how different groups of humans make sense of them at different points in time. In recent decades, however, both the ontological and the epistemological divide between the two cultures of the natural sciences on the one hand and the humanities and interpretive social sciences on the other hand have been called into question from very different angles. Culture is no longer the exclusive domain of the humanities and human sciences but has become an object of research in cultural neuroscience and cultural primatology $(28,29)$. The same is true for related ontological categories such as the mind, which used to organize the disciplinary divide between Geistes- and Naturwissenschaften in the German-speaking world but is now the subject matter of evolutionary and brain research. This new naturalism has inspired research programs in the social sciences that have sought to break down the barrier between the natural and the human by presenting the scientific objects of the natural sciences as constructed by humans (30-32). While these challenges to the dichotomies of nature/culture, nature/human, or mind/matter might not be equally compelling across all areas of study, they are germane to a class of psychoactive drugs whose effects depend on their users' personalities, expectations, and cultural beliefs as well as the social setting in which they are ingested. Studying the moral effects of psychedelics raises epistemological challenges that extend across the divide between the natural sciences and the humanities (33).

More recently, extra-pharmacological factors have attracted attention in psychedelic research, which again stimulates interdisciplinary debates and transdisciplinary collaborations $(34,35)$. The experiential diversity of psychedelic states calls for novel approaches combining phenomenological methods and empirical research to better understand the structure and dynamics of altered states of consciousness that go beyond their conceptualization as "brain states". Social anthropologist David Dupuis, clinical psychologist Rosalind Watts, and neuroscientist Christopher Timmermann propose moving beyond research that correlates first-person reports and third-person measurements of brain activity and attend to the second-person dimension of what they call "psychedelic apprenticeship" (36). Their focus on the social mediation of psychedelic-induced experiences can account for why psychedelics have turned out to be a "double-edged sword" that can either benefit or harm their users-or even do both at the same time. Psychedelics have been shown to increase suggestibility while concealing this influence of others by endowing visions with a noetic quality, a deep sense of having obtained unmediated knowledge that requires no external validation or evidence (37-39). This more variable set of outcomes opens up the possibility of harnessing psychedelic apprenticeships to different therapeutic goals and ethical projects. Subjects undergoing drug-induced mystical experiences might even be steered toward religious conversion and New Age spiritualities-a prospect that has raised concern among psychedelic psychotherapists who seek to secularize the field and worry about the abuse of power on the part of guides and therapists $(40,41)$.

The context sensitivity of psychedelics entails that randomized placebo-controlled trials are insufficient for appreciating the full range of their possible effects, nor do they capture the diverse ways in which consumers may encounter psychedelics in medical and non-medical settings alike. If the pharmacological activity of a drug changes the relationship between a living thing and its environment in ways that depend on the living thing's mindset and the particular quality of the environment, then the environment would be inscribed in the observed pharmacological effect-even if the placebo effects were subtracted from the effects of the verum. In the 1950s, anthropologist Anthony Wallace had already noted that Euro-American subjects who had received mescaline in the laboratory reported very different psychotropic effects than Native Americans who had eaten peyote buttons in religious ceremonies. At a time when placebo-controlled trials only just emerged as the gold standard of pharmacological research, Wallace proposed to complement them by culture-controlled trials that tested the same drug at the same dosage under different social and cultural circumstances [(13), p. 116-26, 18592; (42)]. Reanimating this largely forgotten approach today would provide moral psychopharmacology with an opportunity to import experimental paradigms from social, moral, and political psychology.

\section{"UPSTREAM" DESIGN OF NEW USES OF PSYCHEDELICS}

The ethical plasticity of psychedelic-induced experiences poses a formidable bioethical problem. Usually, bioethics offers a moral evaluation of the social consequences of new biotechnologies or pharmaceuticals. For example, debates over antidepressants or so-called cognitive enhancers assumed that the drugs have certain effects established by clinical observation and psychopharmacological experiment, while bioethicists evaluated the social consequences of these effects downstream from pharmacology and proposed how to regulate the drugs' applications. A growing body of bioethical literature on psychedelics evaluates the use of psychedelics to neurochemically enhance the sense of meaning in psychedelicassisted psychotherapy and their use to develop virtues, suppress vices, and enhance moral behavior $(43,44)$. What distinguishes the notion of psychedelic moral enhancement, as advocated by some authors, from traditional notions is that it recognizes that amplifying particular aspects of prosociality produces trade-offs and adverse effects. For example, MDMA boosts prosocial behavior toward members of one's in-group but not toward the outgroup, which can result in a redistribution of limited resources (45). However, if psychedelics reduced their users' 
sense of self-importance, they might free up resources in favor of both neighbors and strangers, increasing other-regard solely at a cost to egocentric motivations $(46,47)$.

This model of bioethics presupposes conceptions of what counts as moral and measures technologies against them. It is based on a separation of technology and morality, means and ends. However, psychedelics present a case where the means transform the ends. Although a recent trial by Carhart-Harris et al. comparing psilocybin and escitalopram in long-standing, moderate-to-severe major depressive disorder did not show significant difference in antidepressant effects, it produced secondary effects on qualitative dimensions of psychosocial functioning that transformed patients' attitudes toward themselves, others, and the world (48). Notably, patients from other trials undergoing conventional treatment for depression reported that medications and short-term talk therapies tended to reinforce their sense of disconnection and avoidance, whereas psychedelic treatment encouraged connection and acceptance or a sense of "reconnecting" with past values that had faded over the years (49-51). Of course, these experiential reports leave open whether people actually reconnect with their own values, what role their heightened suggestibility plays in this process, and whether the pharmacological modulation of affective relations to the world and to others privileges certain moral and maybe even political transformations over others. Do different psychedelic compounds produce different moral effects and ethical orientations? How do psychedelics compare with other classes of psychotropic drugs? Whatever the mechanism, variability, and scope of such pharmacological transvaluation of values, it led psychiatrist William Smith and medical ethicist Dominic Sisti to call for an enhanced consent process acknowledging that patients might experience significant shifts in their ethical outlook and worldview, which they cannot fully foresee from the perspective of their pre-therapeutic self (52). The challenge is to invent new research practices that observe and reflect on the combining of neurochemistry and morality in the laboratories and clinics where this amalgamation is happening.

Another challenge involves observing how psychedelics are used beyond the laboratory and the clinic, turning psychopharmacology into a field science by adopting practices from ethnopharmacology, ecology, and other areas of field biology. For instance, battery-powered EEG equipment already allows one to study in naturalistic settings how ayahuasca, an Amazonian jungle environment, guidance by experienced ayahuasceros, and other variables concur in their effect on brain waves, and placebo-controlled field experiments seek to tease apart the impact of pharmacological and extra-pharmacological factors in such an ayahuasca ceremony $(53,54)$. Other field sciences such as cultural primatology that use recent advances in statistics to explain how complex interactions between large numbers of ecological factors shape animal behavior might provide further methodological inspiration for how to study the interplay between pharmacological and extrapharmacological factors [e.g., (29, 55), p.180-90; (56)].

Finally, the challenge is to study historical uses of psychedelics as natural experiments that shed light on the context dependence of the psychedelic experience. From a regulatory perspective, it will still largely depend on randomized placebo-controlled trials, whether psychedelics will have a wide or a restricted role as prescription medicines. The goal is not to replace but to complement those trials, as a wide range of contextual variables are likely to determine the therapeutic benefits of psychedelics. An upstream approach to the morally transformative effects of psychedelics places psychopharmacologists, psychiatrists, social researchers, and humanities scholars side by side to study, assess, and redesign psychedelic experiences in their social, cultural, and historical contexts $(57,58)$. Ideally, laboratories and clinical trial sites would embed anthropological, sociological, and philosophical observers instead of keeping them cordoned off in their respective university departments. Ethnographic descriptions and comparisons of the uses of psychedelic drugs at different sites, including non-medical sites, could be fed back into the fashioning of new uses. First-hand familiarity with research and therapeutic practices as well as everyday personal contact enable members of different disciplines to inform each other's thinking and devise approaches that make good use of the context dependence of psychedelics in light of ongoing discussions of what constitutes a good use.

\section{CONCLUSION: EXTENDING RESEARCH AND DEVELOPMENT INTO THE EXTRA-PHARMACOLOGICAL REALM}

If moral psychopharmacology took it upon itself to develop forms of psychedelic apprenticeship for the currently sprawling medical and non-medical applications of psychedelics, it would extend pharmaceutical research and development into the extra-pharmacological realm. Such a design process needs to be informed by best practices in clinical psychology and cognate fields, but, intellectually, it cannot hide behind professional prescriptions because what counts as good and bad is precisely what is at stake here. It is an open philosophical question that has to be answered in a recursive process of psychopharmacological experimentation, clinical and ethnographic observation, historical research, and ethical reflection. The evaluation of new uses of different drugs in the laboratory, the clinic, and in the wild should not be confined to the armchair, removed from these spaces and the experiences they engender. That is why research and development of psychedelics in context also requires research and development of knowledge cultures that bridge the gap between neuropsychopharmacology, social research, and the humanities. It is an empirical question whether such a blending of moral psychopharmacology with moral inquiry would remain confined to psychedelics because of their peculiar pharmacological properties, or whether it could become a model for working with other classes of psychotropic substances as well.

\section{DATA AVAILABILITY STATEMENT}

The raw data supporting the conclusions of this article will be made available by the authors, without undue reservation. 


\section{AUTHOR CONTRIBUTIONS}

NL, DR, MS, and ED contributed to conception and design of the manuscript. NL wrote the first

\section{REFERENCES}

1. Crockett MJ, Clark L, Tabibnia G, Lieberman MD, Robbins TW. Serotonin Modulates Behavioral Reactions to Unfairness. Science. (2008) 320:1739. doi: 10.1126/science.1155577

2. Kosfeld M, Heinrichs M, Zak PJ, Fischbacher U, Fehr E. Oxytocin Increases Trust in Humans. Nature. (2005) 435:673-6. doi: 10.1038/nature 03701

3. Dreu De CKW, Greer LL, Van Kleef GA, Shalvi S, Handgraaf MJJ. Oxytocin promotes human ethnocentrism. Proc Natl Acad Sci USA. (2011) 108:1262-6. doi: $10.1073 /$ pnas. 1015316108

4. Quednow BB. Social cognition and interaction in stimulant use disorders. Curr Opin Behav Sci Addict. (2017) 13:55-62. doi: 10.1016/j.cobeha.2016.10.001

5. Preller KH, Vollenweider FX. Modulation of social cognition via hallucinogens and 'Entactogens.' Front Psychiatry. (2019) 10:881. doi: 10.3389/fpsyt.2019.00881

6. Carhart-Harris RL, Roseman L, Haijen E, Erritzoe D, Watts R, Branchi I, et al. Psychedelics and the essential importance of context: J Psychopharmacol. (2018) 32:725-31. doi: 10.1177/0269881118754710

7. Winkelman M. Psychointegrators: multidisciplinary perspectives on the therapeutic effects of hallucinogens. Complement Health Pract Rev. (2001) 6:219-37. doi: 10.1177/153321010100600304

8. Yaden DB, Griffiths RR. The subjective effects of psychedelics are necessary for their enduring therapeutic effects. ACS Pharmacol Transl Sci. (2021) 4:568-72. doi: 10.1021/acsptsci.0c00194

9. Earp BD, Savulescu J. Love Drugs: The Chemical Future of Relationships. Stanford, CA: Stanford University Press (2020).

10. Forstmann M, Sagioglou C. Lifetime experience with (Classic) psychedelics predicts pro-environmental behavior through an increase in nature relatedness. J Psychopharmacol. (2017) 31:975-88. doi: 10.1177/0269881117714049

11. Forstmann M, Yudkin DA, Prosser AMB, Megan Heller S, Crockett MJ. Transformative experience and social connectedness mediate the moodenhancing effects of psychedelic use in naturalistic settings. Proc Nat Acad Sci USA. (2020) 117:2338-46. doi: 10.1073/pnas.1918477117

12. Earp BD. Psychedelic moral enhancement. R Inst Philos Suppl. (2018) 83:41539. doi: 10.1017/S1358246118000474

13. Langlitz N. Neuropsychedelia: The Revival of Hallucinogen Research since the Decade of the Brain. Berkeley, CA: University of California Press (2012).

14. Furst P. Hallucinogens and Culture. San Francisco, CA: Chandler and Sharp (1976)

15. Myerhoff B. Organization and Ecstasy: Deliberate and Accidental Communitas among Huichol Indians and American Youth. In: Moore SF, Myerhoff B, editors. Symbol and Politics in Communal Ideology. Cases and Questions. Ithaca, NY: Cornell University Press (1976).

16. Dawson AS. The Peyote Effect: From the Inquisition to the War on Drugs. Oakland: University of California Press (2018).

17. Dyck E. Peyote and psychedelics on the canadian prairies. In: Caiuby Labate B, Cavnar C, editors. Peyote: History, Tradition, Politics, and Conservation. Santa Barbara, CA: Praeger (2016). p. 151-70.

18. Blum R. Utopiates. The Use and Users of LSD-25. New York, NY: Atherton (1964).

19. Dobkin de Rios M. Hallucinogens. Cross-Cultural Perspectives. Prospect Heights, IL: Waveland Press (1984).

20. Taylor AC. Remembering to forget: identity, mourning and memory among the jivaro. Man. (1993) 28:653-78. doi: 10.2307/2803991

21. Powers N. Black masks, rainbow bodies: psychedelics and race. MAPS Bulletin. (2017) 28:47-51. draft of the manuscript. DR, MS, and ED wrote sections of the manuscript. All authors contributed to manuscript revision, read, and approved the submitted version.

22. Williams MT, Reed S, George J. Culture and psychedelic psychotherapy: ethnic and racial themes from three black women therapists. J Psychedelic Stud. (2020) 4:125-38. doi: 10.1556/2054.2020.00137

23. Nour MM, Evans L, Carhart-Harris RL. Psychedelics, personality and political perspectives. J Psychoactive Drugs. (2017) 49:182-91. doi: 10.1080/02791072.2017.1312643

24. Johnson MW, Yaden DB. There's no good evidence that psychedelics can change your politics or religion. Sci Am. (2020) 5:2020.

25. Langlitz N. Rightist psychedelia. Cult Anthropol Hot Spots. (2020) Available online at: https://culanth.org/fieldsights/rightist-psychedelia? $\mathrm{x}$-craft-preview=05DZPjgCnc\&token=ZqYAgoBfV3OMGpVfwU-wTlBbg OzqUGKA (accessed July 26, 2020).

26. Piper A. Strange Drugs Make For Strange Bedfellows: Ernst Jünger, Albert Hofmann and the Politics of Psychedelics. Portland, OR: Invisible College Publishing (2015).

27. Makuch B, Lamoureux M. Neo-Nazi memoir describes terror group's acid-soaked ram sacrifice. VICE Magazine (2020, June 24). Available online at: https://www.vice.com/en/article/z3e3ew/neo-nazi-memoir-describesterror-groups-acid-soaked-ram-sacrifice

28. Chiao JY. Cultural neuroscience: a once and future discipline. Prog Brain Res. (2009) 178:287-304. doi: 10.1016/S0079-6123(09)17821-4

29. Langlitz N. Chimpanzee Culture Wars: Rethinking Human Nature alongside Japanese, European, and American Cultural Primatologists. Princeton, NJ: Princeton University Press (2020).

30. Latour B. Science in Action: How to Follow Scientists and Engineers Through Society. Cambridge, MA: Harvard University Press (1987).

31. Rheinberger H-J. Toward a History of Epistemic Things: Synthesizing Proteins in the Test Tube. Stanford: Stanford University Press (1997).

32. Helmreich S. Waves: an anthropology of scientific things. HAU J EthnogrTheory. (2014) 4:265-84. doi: 10.14318/hau4.3.016

33. Langlitz N. Delirious brain chemistry and controlled culture: exploring the contextual mediation of drug effects. In: Choudhury S, Slaby J, editors. Critical Neuroscience: A Handbook of the Social and Cultural Contexts of Neuroscience. London: Wiley (2012). p. 253-62.

34. Langlitz N. Kultivierte Neurochemie und unkontrollierte Kultur. [Debate with Bajbouj, M, Quednow, B, Jäger, L.]. Zeitschrift für Kulturwissenschaften. (2010) 2:61-88.

35. Hartogsohn I. Set and setting, psychedelics and the placebo response: an extrapharmacological perspective on psychopharmacology. J Psychopharmacol. (2016) 30:1259-67. doi: 10.1177/0269881116677852

36. Timmermann C, Watts R, Dupuis D. Towards psychedelic apprenticeship: developing a gentle touch for the mediation and validation of psychedelic-induced insights and revelations. Transcult Psychiatry. (2021). doi: 10.31234/osf.io/j5768. [Epub ahead of print].

37. Carhart-Harris RL, Kaelen M, Whalley MG, Bolstridge M, Feilding A, Nutt DJ. LSD enhances suggestibility in healthy volunteers. Psychopharmacology. (2015) 232:785-94. doi: 10.1007/s00213-014-3714-z

38. Pahnke W, Richards W. Implications of LSD and experimental mysticism. J Relig Health. (1966) 5:175-208. doi: 10.1007/BF01532646

39. Preller KH, Herdener M, Pokorny T, Planzer A, Kraehenmann R, Stämpfli $\mathrm{P}$, et al. The fabric of meaning and subjective effects in LSD-induced states depend on serotonin 2A receptor activation. Curr Biol. (2017) 27:451-7. doi: $10.1016 /$ j.cub.2016.12.030

40. Dupuis D. The socialization of hallucinations: cultural priors, social interactions and contextual factors in the use of psychedelics. Transcult Psychiatry. (In press).

41. Johnson MW. Consciousness, religion, and gurus: pitfalls of psychedelic medicine. ACS Pharmacol Transl Sci. (2021) 4:578-81. doi: $10.1021 /$ acsptsci.0c0019 
42. Wallace AFC. Cultural determinants of response to hallucinatory experience. Arch Gen Psychiatry. (1959) 1:58-69. doi: 10.1001/archpsyc.1959.03590010074009

43. Hughes JJ. Using neurotechnologies to develop virtues: a buddhist approach to cognitive enhancement. Account Res. (2013) 20:27-41. doi: 10.1080/08989621.2013.749744

44. McMillan RM. Prescribing meaning: hedonistic perspectives on the therapeutic use of psychedelic-assisted meaning enhancement. J Med Ethics. (2020). doi: 10.1136/medethics-2020-106619. [Epub ahead of print].

45. Kirkpatrick M, Delton AW, Robertson TE, de Wit H. Prosocial effects of MDMA: a measure of generosity. J Psychopharmacol. (2015) 29:661-8. doi: $10.1177 / 0269881115573806$

46. Ahlskog R. Moral enhancement should target self-interest and cognitive capacity. Neuroethics. (2017) 10:363-73. doi: 10.1007/s12152-017-9331-x

47. Kähönen J. Psychedelic Unselfing and Moral Perception : A Philosophical Account of the Change of Values Induced by Psychedelic Experiences. (thesis MA). Helsinki: University of Helsinki (2020).

48. Carhart-Harris RL, Giribaldi B, Watts R, Baker-Jones M, Murphy-Beiner A, Murphy R, et al. Trial of psilocybin versus escitalopram for depression. N Eng J Med. (2021) 384:1402-11. doi: 10.1056/NEJMoa2032994

49. Carhart-Harris RL, Erritzoe D, Haijen E, Kaelen M, Watts R. Psychedelics and connectedness. Psychopharmacology. (2018) 235:547-50. doi: 10.1007/s00213-017-4701-y

50. Noorani T, Garcia-Romeu A, Swift TC, Griffiths RR, Johnson MW. Psychedelic therapy for smoking cessation: qualitative analysis of participant accounts. J Psychopharmacol. (2018) 32:756-69. doi: 10.1177/0269881118780612

51. Watts R, Day C, Krzanowski J, Nutt D, Carhart-Harris RL. Patients' accounts of increased 'connectedness' and 'acceptance' after psilocybin for treatment-resistant depression. J Humanist Psychol. (2017) doi: 10.1177/0022167817709585

52. Smith WR, Sisti D. Ethics and ego dissolution: the case of psilocybin. J Med Ethics. (2020). doi: 10.1136/medethics-2020-106070. [Epub ahead of print].

53. Hoffmann E, Keppel Hesselink JM, da Silveira Barbosa Y. Effects of a psychedelic, tropical tea, ayahuasca, on the electroencephalographic (EEG) activity of the human brain during a shamanistic ritual. MAPS Bull. (2001) 11:25-30.

54. Uthaug MV, Mason NL, Toennes SW, Reckweg JT, de Sousa Fernandes Perna EB, et al. A placebo-controlled study of the effects of ayahuasca, set and setting on mental health of participants in ayahuasca group retreats. Psychopharmacology. (2021) 238:1899-910. doi: 10.1007/s00213021-05817-8

55. Luncz LV, Mundry R, Boesch C. Evidence for cultural differences between neighboring chimpanzee communities. Curr Biol. (2012) 22:922-6. doi: 10.1016/j.cub.2012.03.031

56. Shipley B. Cause and Correlation in Biology: A User's Guide to Path Analysis, Structural Equations and Causal Inference With R. Cambridge, UK: Cambridge University Press (2000).

57. Earp BD, Yaden DB. Culture, context, and community in contemporary psychedelic research. Philos Psychiatry Psychol. (In press).

58. Rabinow P, Bennett G. Designing Human Practices: An Experiment With Synthetic Biology. Chicago: University of Chicago Press (2012).

Conflict of Interest: The authors declare that the research was conducted in the absence of any commercial or financial relationships that could be construed as a potential conflict of interest.

Publisher's Note: All claims expressed in this article are solely those of the authors and do not necessarily represent those of their affiliated organizations, or those of the publisher, the editors and the reviewers. Any product that may be evaluated in this article, or claim that may be made by its manufacturer, is not guaranteed or endorsed by the publisher.

Copyright (C) 2021 Langlitz, Dyck, Scheidegger and Repantis. This is an open-access article distributed under the terms of the Creative Commons Attribution License (CC $B Y)$. The use, distribution or reproduction in other forums is permitted, provided the original author(s) and the copyright owner(s) are credited and that the original publication in this journal is cited, in accordance with accepted academic practice. No use, distribution or reproduction is permitted which does not comply with these terms. 